\title{
Comparative cost-effectiveness of stereotactic body radiation therapy versus intensity-modulated and proton radiation therapy for localized prostate cancer
}

\section{Anju Parthan ${ }^{1 *}$, Narin Pruttivarasin ${ }^{1}$, Diane Davies ${ }^{2}$, Douglas C. A. Taylor ${ }^{1}$, Vivek Pawar ${ }^{3}$, Akash Bijlani ${ }^{2}$, Kristen Hassmiller Lich ${ }^{4}$ and Ronald C. Chen ${ }^{4}$}

${ }^{1}$ Health Economics and Outcomes Research, OptumInsight

${ }^{2}$ Accuray Incorporated

${ }^{3}$ Bayer (Formerly with Optumlnsight)

${ }^{4}$ University of North Carolina at Chapel Hill

Edited by:

Brian Timothy Collins, Georgetown

University Hospital, USA

Reviewed by:

Sean Collins, Georgetown University

Hospital, USA

Debra Freeman, Naples Radiation

Oncology, USA

${ }^{*}$ Correspondence:

Anju Parthan, Health Economics and

Outcomes Research, Optum/nsight,

425 Market Street, 12th Floor, San

Francisco, CA 94105, USA.

e-mail: anju.parthan@optum.com
Objective: To determine the cost-effectiveness of several external beam radiation treatment modalities for the treatment of patients with localized prostate cancer. Methods: A lifetime Markov model incorporated the probabilities of experiencing treatment-related long-term toxicity or death. Toxicity probabilities were derived from published sources using meta-analytical techniques. Utilities and costs in the model were obtained from publicly available secondary sources. The model calculated quality-adjusted life expectancy and expected lifetime cost per patient, and derived ratios of incremental cost per qualityadjusted life year (QALY) gained between treatments. Analyses were conducted from both payer and societal perspectives. One-way and probabilistic sensitivity analyses were performed. Results: Compared to intensity-modulated radiation therapy (IMRT) and proton beam therapy (PT), stereotactic body radiation therapy (SBRT) was less costly and resulted in more QALYs. Sensitivity analyses showed that the conclusions in the base-case scenario were robust with respect to variations in toxicity and cost parameters consistent with available evidence. At a threshold of \$50,000/QALY, SBRT was cost-effective in 75\% and $94 \%$ of probabilistic simulations compared to IMRT and PT, respectively, from a payer perspective. From a societal perspective, SBRT was cost-effective in $75 \%$ and $96 \%$ of simulations compared to IMRT and PT, respectively, at a threshold of $\$ 50,000 / \mathrm{QALY}$. In threshold analyses, SBRT was less expensive with better outcomes compared to IMRT at toxicity rates $23 \%$ greater than the SBRT base-case rates. Conclusion: Based on the assumption that each treatment modality results in equivalent long-term efficacy, SBRT is a cost-effective strategy resulting in improved quality-adjusted survival compared to IMRT and PT for the treatment of localized prostate cancer.

Keywords: prostate, cancer, radiation, cost-effectiveness, localized

\section{INTRODUCTION}

The National Cancer Institute estimates that 241,740 new cases of prostate cancer will be diagnosed in 2012, along with 28,170 deaths (National Cancer Institute, 2012). The Prostate Cancer Outcomes Study (PCOS), which was initiated by the National Cancer Institute in 1994, noted that $88 \%$ of the newly diagnosed prostate cancer cases will be localized disease. Based on a review of the current published literature, there are several options for patients diagnosed with clinically localized prostate cancer including active surveillance, surgery, and radiation therapy. In 2010, the Agency for Healthcare Research and Quality (AHRQ) concluded that insufficient evidence exists to fully assess the superiority of one type of radiation therapy over another for the treatment of localized prostate cancer (Agency for Healthcare Research and Quality, 2010). The objective of this study is to address this gap by developing a decision analysis model that integrates currently available published evidence on the post-treatment incidence of long-term toxicities to compare the incremental cost-effectiveness of three modern external beam radiation therapies for localized prostate cancer: intensity-modulated radiation therapy (IMRT), proton beam therapy (PT), and stereotactic body radiation therapy (SBRT).

Intensity-modulated radiation therapy, which is a commonly used treatment for patients with localized prostate cancer, involves the external delivery of multiple beams of radiation that conform to the shape of the tumor, and where the intensity of each beam can be modulated in order to spare surrounding healthy tissue. IMRT therapy is typically delivered in 40 fractions (i.e., treatment sessions) and requires $8-9$ weeks of treatment. PT is delivered externally to a predefined depth, potentially with little radiation delivered beyond that point, thus sparing surrounding healthy tissue. Like IMRT, PT typically is delivered in 40 fractions and requires 8-9 weeks of treatment. A study conducted by Konski et al. (2007) found that PT was not a cost-effective 
treatment option for localized prostate cancer when compared to IMRT.

Stereotactic body radiation therapy is the use of accurate and image-guided radiation therapy to treat tumors using up to five intense radiation treatments (Martin and Gaya, 2010). Because a higher dose is given in each fraction, image guidance during treatment and an ability to adjust for tumor/target motion is important in order to minimize treatment-related toxicity. Recent studies have indicated that SBRT toxicity levels are comparable to those of other radiation treatment options (Sanda et al., 2008; Chen et al., 2009; Townsend et al., 2011), with long-term (5-year) progression-free survival at 93\% (Freeman and King, 2011).

In the current paper we present a Markov model developed to estimate the comparative long-term incremental cost-effectiveness of IMRT, PT, and SBRT. The analyses are conducted using a lifetime horizon from both the perspective of a health care payer as well as a societal perspective that includes the value of patients' time spent in treatment.

\section{MATERIALS AND METHODS OVERVIEW}

Decision-analytic techniques involved analysis of a lifetime cohort Markov model, programmed in Microsoft ${ }^{\circledR}$ Excel, to assess the cost-effectiveness of three radiation treatment modalities for localized prostate cancer: SBRT, IMRT, and PT. Brachytherapy was not included as a comparator in the model because the method of delivery (i.e., requiring anesthesia and invasive) was considered to be significantly different from SBRT, IMRT, and PT. Furthermore, because of the anesthesia requirement, some studies have shown that brachytherapy is more likely to be used with younger men when compared with external beam radiation (Sanda et al., 2008; Chen et al., 2009).

Depending on the treatment selected, patients in the model are at risk of experiencing one or more of three types of treatmentrelated long-term toxicity, or death. Long-term toxicities included in the analysis were gastrointestinal (GI), genitourinary (GU), and sexual dysfunction (SD). Long-term toxicity is defined as adverse events $=$ grade 2, using the Radiation Therapy Oncology Group (RTOG) scale $\geq 12$ months following treatment. The model assumes that patients can experience treatment-related long-term toxicity within the first year following treatment and are at risk to continue to experience it throughout their lives. Figure 1 illustrates the Markov states and transitions that can occur in the model. The model includes eight health states that reflect all possible combinations of the three long-term toxicity states (no toxicity, GI only, GU only, SD only, GI \& GU, GI \& SD, GU \& SD, GI \& GU \& SD). Since data were not available regarding the duration of side effects, the assumption in the model is that during each Markov cycle patients remain in the same Markov health state or die. Measures of utility (an overall quality of life measure on a 0 to 1 scale) and cost are assigned to each state. Treatment-specific mortality and long-term annual mortality rates with localized prostate cancer are based on published sources and data from SEER, respectively. The model assumes that the long-term disease control is comparable across the treatments, which is consistent with currently available literature.

In the base-case we assume that all toxicities are associated with a one-time cost. Analysis of quality-adjusted survival with the Markov model involves tracking patients according to whether or not they experience each of the types of long-term toxicity, and weighting their years of survival by the utility associated with their corresponding toxicity state. The model calculates the qualityadjusted life years (QALYs) and costs per patient over the course of a lifetime. Analyses were performed from a healthcare payer perspective. An additional analysis was conducted from a societal perspective that included the forgone value of patients' time spent in initial radiation treatment.

\section{MODEL PARAMETERS AND DATA SOURCES}

The model population consists of 65-year-old males with localized prostate cancer who declined or were ineligible for surgery and are eligible for external radiation therapies. Model parameters and data sources are summarized in Tables 1-5, and are described below.

\section{Long-term toxicity and mortality}

The probability of experiencing each type of treatment-related long-term toxicity was derived using meta-analytic techniques.

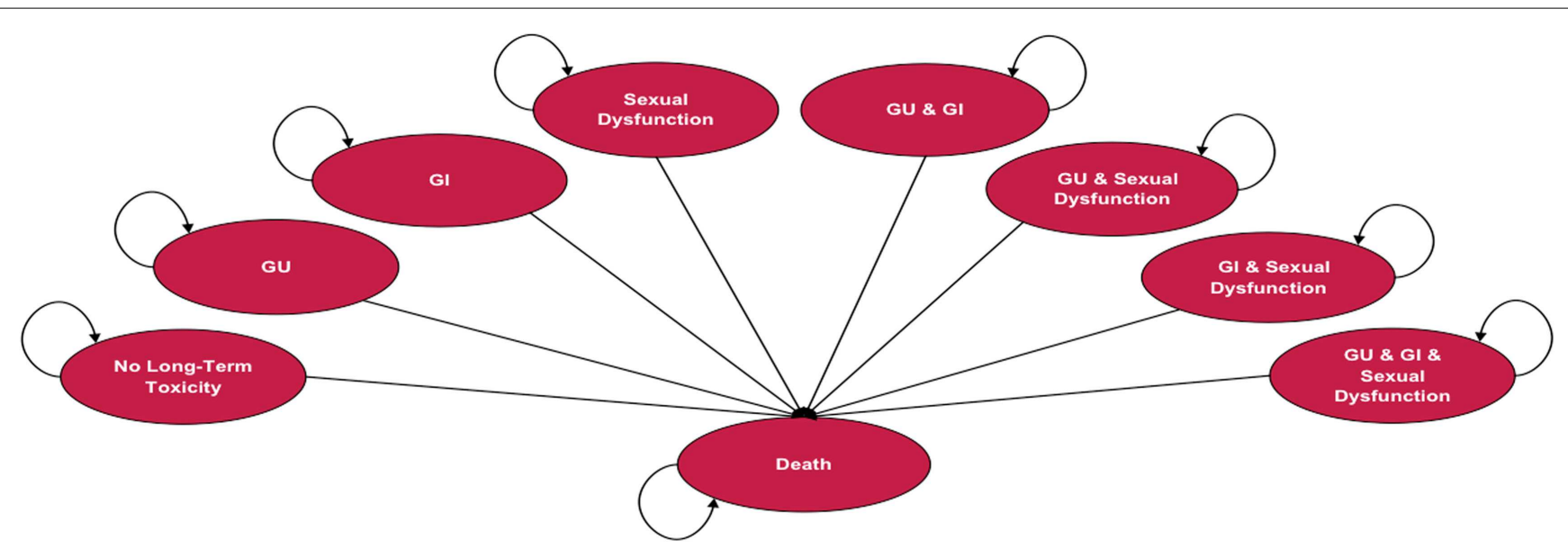

FIGURE 1 | Markov model. GI, gastrointestinal; GU, genitourinary; SD, sexual dysfunction. 
A search was conducted in MEDLINE, EMBASE, PsycINFO, and PubMed for studies published between 1998 and 2010 that reported GU, GI, and SD toxicities over a given period of time. Table 1 displays the characteristics of the 10 studies that were chosen for the meta-analysis. Due to a lack of randomized controlled trials, all 10 studies were either clinical trials with no control group or observational studies with a single cohort. The meta-analysis used a random-effects model to minimize heterogeneity across studies due to clinical or methodological differences. Furthermore, the meta-analysis used the normally distributed log odds ratio as the outcome variable, after which the pooled log odds ratio was converted back to an annual rate.

Table 2 displays the treatment-related probabilities of experiencing the various types of long-term toxicity and mortality. A recent paper published by Coen et al. (2012) suggests that PT toxicity has changed very little in the last 10 years. Also, in the absence of long-term SD toxicity rates for PT, in the base-case, the SD rates were assumed to be equal to IMRT because the method of delivery over time was similar - i.e., small doses over a long period of time. An age-specific mortality rate for prostate cancer patients was applied to all patients in the model using SEER data (Altekruse et al., 2010). The risk of dying from treatment (IMRT, SBRT, and PT) was assumed to be zero.

\section{Health-related quality of life}

Quality-adjusted life expectancy associated with each treatment was estimated using utilities assigned to each health state in the model (Table 3). Utilities are weights that quantify health-related

Table 1 | Characteristics of the 10 studies chosen for the meta-analysis.

\begin{tabular}{|c|c|c|c|c|c|}
\hline Treatment & Study & Sample size & $\begin{array}{l}\text { Follow-up } \\
\text { time (months) }\end{array}$ & Age (range) & Toxicity studied \\
\hline \multirow[t]{5}{*}{ SBRT } & Friedland et al. (2009) & 112 & 24 & Mean 70 (55-87) & SD \\
\hline & Katz et al. (2010) & 206 & 17 (dose 36.25) & Mean 69 (45-88) & GU, GI, SD \\
\hline & & & 30 (dose 35) & & \\
\hline & King et al. (2009) & 41 & 33 & Median 66 (48-83) & GU, GI \\
\hline & Wiegner and King (2010) & 20 & 35.5 & Median 68 (57-83) & $\mathrm{SD}$ \\
\hline \multirow[t]{3}{*}{ IMRT } & Kirichenko et al. (2006) & 928 & 36 & NA & $\mathrm{GU}, \mathrm{GI}$ \\
\hline & Zelefsky et al. (2002) & 772 & 36 & Median 69 (46-86) & GU, GI, SD \\
\hline & Zelefsky et al. (2006) & 561 & 96 & Median 68 (46-86) & GU, GI, SD \\
\hline \multirow[t]{3}{*}{ PT } & Schulte et al. (2000) & 870 & 36 & NA & GU, GI \\
\hline & Slater et al. (1998) & 643 & 36 & NA & $\mathrm{GU}, \mathrm{GI}$ \\
\hline & Slater et al. (1999) & 315 & 36 & NA & $\mathrm{GU}, \mathrm{Gl}$ \\
\hline
\end{tabular}

GI, gastrointestinal; GU, genitourinary; IMRT, intensity-modulated radiation therapy; NA, not available; PT, proton beam therapy; SBRT, stereotactic body radiation therapy; SD, sexual dysfunction.

Table 2 | Treatment-related mortality and long-term toxicity.

\begin{tabular}{llll}
\hline Probabilities & \multicolumn{1}{c}{ Base-case default value } & SE & Source \\
\hline TREATMENT-RELATED & MORTALITY & & \\
\hline SBRT & 0.000 & - & Assumption \\
IMRT & 0.000 & - & Assumption \\
PT & 0.000 & - & Assumption \\
LONG-TERM TOXICITIES - SBRT & & \\
GU & 0.040 & 0.023 & Friedland et al. (2009), Katz et al. (2010), King et al. (2009) \\
GI & 0.027 & 0.010 & Katz et al. (2010), King et al. (2009) \\
SD & 0.159 & 0.088 & Friedland et al. (2009), Katz et al. (2010), Wiegner and King (2010) \\
LONG-TERM TOXICITIES - IMRT & & \\
GU & 0.035 & 0.016 & Kirichenko et al. (2006), Zelefsky et al. (2002), Zelefsky et al. (2006) \\
GI & 0.013 & 0.008 & Kirichenko et al. (2006), Zelefsky et al. (2002), Zelefsky et al. (2006) \\
SD & 0.272 & 0.232 & Zelefsky et al. (2002), Zelefsky et al. (2006) \\
LONG-TERM TOXICITIES - PT & & \\
GU & 0.019 & & Schulte et al. (2000), Slater et al. (1998), Slater et al. (1999) \\
GI & 0.015 & 0.004 & Schulte et al. (2000), Slater et al. (1999) \\
SD & 0.272 & 0.006 & Assume the same as IMRT
\end{tabular}

GI, gastrointestinal; GU, genitourinary; IMRT, intensity-modulated radiation therapy; PT, proton beam therapy; SBRT stereotactic body radiation therapy; SD, sexual dysfunction; SE, standard error.

An RTOG (Radiation Therapy Oncology Group) scale was used to measure toxicity in all but the Kirichenko et al., 2006; scale not reported) and Zelefsky et al., 2006; CTCAE scale) studies. 
quality of life (HRQoL) on a scale from 0 to 1 , where 0 reflects death and 1 reflects perfect health. The QALYs associated with each of the long-term toxicity health states were estimated using utility weights derived from Stewart et al. (2005). The utility value for not experiencing treatment-related long-term toxicity was assumed to be 1 , and the utility value for death was 0 . QALYs in each Markov state and cycle were determined by multiplying the utility

Table 3 | Model utility inputs.

\begin{tabular}{llll}
\hline Utility parameters & $\begin{array}{l}\text { Base-case } \\
\text { default value }\end{array}$ & SE & Source \\
\hline No long-term toxicities & 1.00 & 0.00 & Assumption \\
GU & 0.83 & 0.02 & Stewart et al. (2005) \\
GI & 0.71 & 0.02 & Stewart et al. (2005) \\
SD & 0.89 & 0.01 & Stewart et al. (2005) \\
GU \& GI & 0.70 & 0.04 & Stewart et al. (2005) \\
GU \& SD & 0.79 & 0.03 & Stewart et al. (2005) \\
GI \& SD & 0.57 & 0.04 & Stewart et al. (2005) \\
GU \& GI \& SD & 0.45 & 0.04 & Stewart et al. (2005) \\
Death & 0.00 & 0.00 & By definition \\
\hline
\end{tabular}

GI, gastrointestinal; GU, genitourinary; SD, sexual dysfunction; SE, standard error; QALYs in each Markov state and cycle are determined by the product of the baseline age-specific background utility for males (Hamner et al., 2006) and the utility associated with long-term toxicities. associated with each of the long-term toxicities by a baseline agespecific background utility for males in the general population (Hamner et al., 2006).

\section{Resource utilization}

Table 4 displays the base-case resource utilization parameter values for each treatment, including the numbers of routine and toxicity-related office visits and treatments, and the number of patient hours lost due to treatment. The resource utilization parameter values were used to estimate treatment-related costs in the model.

\section{Costs}

Table 5 displays the unit costs per unit of resource utilization that were used in the model. All unit costs in the table reflect Medicare payments. The SBRT treatment cost was based on nationally unadjusted Medicare rates using codes for robotic SBRT, and the remaining treatment costs were based on published sources. For the societal perspective, the age-specific cost per hour of time lost in treatment was based on 2011 estimates from the Bureau of Labor Statistics (2011). Additional patient costs, such as transportation costs, are not included in the societal perspective. From a lost work time perspective, we assumed that this wage value reflects the opportunity cost of time for both working and retired persons of the same age. The cost of routine monitoring and toxicityrelated costs was applied annually for GU and SD whereas for GI a one-time cost was applied assuming that GI symptoms are less

Table 4 | Model resource utilization inputs.

\begin{tabular}{|c|c|c|}
\hline Utilization parameters & Value & Source \\
\hline \multicolumn{3}{|l|}{ TREATMENT-RELATED (PER TREATMENT) } \\
\hline \multicolumn{3}{|l|}{ SBRT } \\
\hline Work-time lost (hours per treatment) & 10 & Expert opinion \\
\hline \multicolumn{3}{|l|}{ IMRT } \\
\hline Work-time lost (hours per treatment) & 90 & Ollendorf et al. (2009) \\
\hline \multicolumn{3}{|l|}{ PT } \\
\hline Work-time lost (hours per treatment) & 100 & Ollendorf et al. (2008) \\
\hline \multicolumn{3}{|l|}{ ROUTINE MONITORING (PER YEAR) } \\
\hline Office visit (1st year) & 2 & Expert opinion \\
\hline Office visit (subsequent years) & 1 & Expert opinion \\
\hline PSA & 2 & Expert opinion \\
\hline \multicolumn{3}{|l|}{ TOXICITY-RELATED } \\
\hline \multicolumn{3}{|l|}{ GU toxicity (per year) } \\
\hline Routine office visit & 2 & Expert opinion \\
\hline Pharmacologic treatment (oxybutynin) & 365 & Daily dosage based on package insert \\
\hline Cystoscopy & 1 & Expert opinion \\
\hline \multicolumn{3}{|l|}{ GI toxicity (one-time utilization) } \\
\hline Routine office visit & 2 & Assumption \\
\hline Enema (additional colonoscopy and sigmoidoscopy if needed)* & 1 & Assume one-time treatment for GI toxicity \\
\hline \multicolumn{3}{|l|}{ SD toxicity (per year) } \\
\hline Pharmacologic treatment (sildenafil) & 22 & Cooke et al. (2005) \\
\hline
\end{tabular}

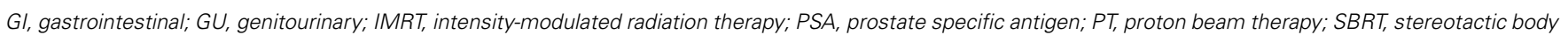
radiation therapy; $S D$, sexual dysfunction.

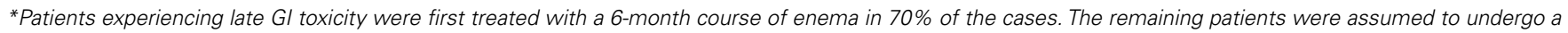

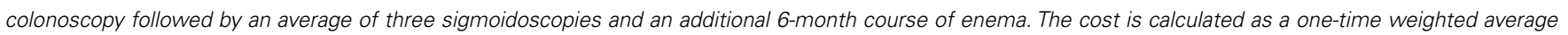
cost. 
Table 5 | Model cost inputs.

\begin{tabular}{|c|c|c|}
\hline Cost parameters & Value & Source \\
\hline \multicolumn{3}{|l|}{ TREATMENT COST } \\
\hline SBRT & $\$ 20,889$ & Medicare rates - data on file, Accuray Inc. \\
\hline PT & $\$ 65,250$ & Ollendorf et al. (2008) \\
\hline Work-time lost (cost per hour) & $\$ 20$ & Bureau of Labor Statistics (2011) \\
\hline Office visit & $\$ 102$ & MAG Mutual (2011) \\
\hline PSA & $\$ 103$ & MAG Mutual (2011) \\
\hline \multicolumn{3}{|l|}{ TOXICITY-RELATED } \\
\hline \multicolumn{3}{|l|}{ GU toxicity } \\
\hline Daily pharmacologic treatment (oxybutynin) & $\$ 1$ & Red Book $(2010)^{\ddagger}$ \\
\hline Daily pharmacologic treatment (sildenafil) & $\$ 12$ & Red Book $(2010)^{\ddagger}$ \\
\hline
\end{tabular}

GI, gastrointestinal; GU, genitourinary; IMRT, intensity-modulated radiation therapy; PSA, prostate specific antigen; PT, proton beam therapy; SBRT, stereotactic body radiation therapy; $S D$, sexual dysfunction.

*Patients experiencing late GI toxicity were first treated with a 6-month course of enema in $70 \%$ of the cases. The remaining patients were assumed to undergo a colonoscopy followed by an average of three sigmoidoscopies and an additional 6-month course of enema. The cost is calculated as a one-time weighted average cost.

${ }^{\dagger}$ Assumes one-time treatment cost.

${ }^{*}$ Costs are inflated to 2011 values based on the Consumer Price Index (Bureau of Labor Statistics, 2011).

likely to be chronic. All costs were based on published sources and costs used in the analysis are expressed in 2011 US dollars, with costs and utilities discounted at $3.0 \%$ annually.

\section{ANALYSES}

\section{Base-case model}

Lifetime costs and QALYs per patient were calculated for each treatment. Lifetime costs were calculated by summing all costs associated with each health state that patients spend time in, and QALYs are calculated by summing the product of utilities associated with each health state and the time spent in the health state. The incremental cost-effectiveness ratio (ICER) value was calculated by first rank-ordering the treatment regimens by increasing cost and then comparing each strategy to the next less costly strategy by dividing the additional cost by additional benefit (QALY).

\section{Sensitivity analyses}

One-way sensitivity analysis was conducted to determine the parameters to which the ICER is most sensitive. One-way sensitivity analyses were performed by varying selected model parameters based on the $95 \%$ confidence interval (CI) of the base-case estimate, where available, while keeping all other parameters constant. CIs were not available for the costs used in the model, and so they were varied through a range of $75 \%-125 \%$ of the base-case estimate while keeping all other parameters constant. Probabilistic sensitivity analysis was also conducted to assess uncertainty in the cost-effectiveness analysis by determining the probability of a treatment being cost-effective at a threshold of \$50,000/QALY.
The probability estimates for toxicity and utility were assumed to follow a beta distribution [see Tables $\mathbf{2}$ and $\mathbf{3}$ for the standard errors (SE)], and cost parameters were assumed to follow a gamma distribution and the SE was calculated as $50 \%$ of the base-case default value.

An alternative cost scenario was tested by applying an annual cost for treating GI toxicity, instead of the one-time costs used in the base-case. In the absence of a SD toxicity rate for PT, an alternative toxicity scenario was tested by setting the SD toxicity for PT equal to SBRT. Finally, in threshold analyses, the toxicity of SBRT was varied from the base-case assumption to assess the impact of alternate toxicity rates on base-case results.

\section{RESULTS}

\section{BASE-CASE MODEL}

Table 6 displays the results of the cost-effectiveness analyses from the payer and societal perspectives.

\section{Payer perspective}

SBRT was the least expensive option in terms of lifetime costs $(\$ 24,873)$, followed by IMRT $(\$ 33,068)$ and PT $(\$ 69,412)$. IMRT and PT were both more costly and yielded fewer QALYs when compared with SBRT (total QALYs: 8.11, 8.05, 8.06 for SBRT, IMRT, $\mathrm{PT}$, respectively).

\section{Societal perspective}

When the value of lost time in treatment was included, SBRT remained the least expensive treatment option $(\$ 25,097)$, followed by IMRT $(\$ 35,088)$ and then PT $(\$ 71,657)$. Once again, IMRT and 
Table 6 | Cost-effectiveness results for all comparator treatments for the base-case.

\begin{tabular}{|c|c|c|c|c|c|}
\hline & \multicolumn{2}{|c|}{ Total per patient } & \multicolumn{2}{|c|}{ Incremental } & \multirow{2}{*}{$\begin{array}{l}\text { ICER Costs/ } \\
\text { QALY gained }\end{array}$} \\
\hline & Costs & QALYs & Costs & QALYs & \\
\hline \multicolumn{6}{|c|}{ PAYER PERSPECTIVE } \\
\hline SBRT & $\$ 24,873$ & 8.11 & - & - & Reference \\
\hline IMRT & $\$ 33,068$ & 8.05 & $\$ 8,195$ & -0.062 & Dominated* \\
\hline PT & $\$ 69,412$ & 8.06 & $\$ 44,539$ & -0.047 & Dominated \\
\hline \multicolumn{6}{|c|}{ SOCIETAL PERSPECTIVE ${ }^{\dagger}$} \\
\hline SBRT & $\$ 25,097$ & 8.11 & - & - & Reference \\
\hline IMRT & $\$ 35,088$ & 8.05 & $\$ 9,991$ & -0.062 & Dominated \\
\hline PT & $\$ 71,657$ & 8.06 & $\$ 46,560$ & -0.047 & Dominated \\
\hline
\end{tabular}

ICER, incremental cost-effectiveness ratio; IMRT, intensity-modulated radiation therapy; $Q A L Y$, quality-adjusted life year.

NOTE: Results are estimated for a patient 65 years old using Medicare reimbursement rates.

*Dominated - higher cost and lower QALY.

${ }^{+}$Societal perspective also includes productivity costs owing to time spent in treatment.

PT were both dominated by SBRT because they were more costly and yielded fewer QALYs when compared with SBRT (total QALYs: 8.11, 8.05, 8.06 for SBRT, IMRT, PT, respectively).

\section{SENSITIVITY ANALYSES}

When the toxicity parameters were varied based on their CIs, and the costs by $\pm 25 \%$, the results did not change from the base-case in both payer and societal perspectives; SBRT was the dominant strategy, being less expensive with more QALYs compared to IMRT and PT. The probabilities of SBRT being cost-effective at \$50,000/QALY from both the payer and societal perspective are presented in Table 7. The probabilities of SBRT being cost-effective compared to IMRT and PT are $75.1 \%$ and $94.1 \%$, respectively, from the payer perspective; $75.1 \%$ and $95.5 \%$, respectively, from the societal perspective. Figure 2 displays the probabilities of SBRT being cost-effective compared to IMRT and PT, from the payer (Figure 2A) and societal (Figure 2B) perspective.

The alternative cost scenario analysis using an annual cost for treating GI toxicity, instead of the one-time costs used in the basecase model, produced results that were similar to the base-case. Both IMRT and PT remained more costly and yielded fewer QALYs when compared with SBRT from either perspective. Also, Table 8 displays the results of an alternative toxicity scenario where SD toxicity for PT was set equal to SBRT, in contrast to the base-case model which set PT equal to IMRT. When the SD toxicity for PT was set equal to SBRT, then SBRT weakly dominates IMRT and no longer dominates PT.

In threshold analyses, when all three toxicity rates for SBRT were increased by $23 \%$ from the base-case, the total lifetime costs for SBRT was lower than for IMRT ( $\$ 25,037$ versus $\$ 33,068)$, and the QALYs for SBRT were slightly higher than for IMRT (8.051 versus 8.049). If all three toxicity rates for SBRT were increased by $\geq 24 \%$, the QALYs for SBRT are lower than for IMRT. Furthermore, the threshold analyses showed that IMRT was only cost-effective
Table 7 | Probability of SBRT being cost-effective at the $\$ 50,000 / Q A L Y$ threshold.

\begin{tabular}{lll}
\hline SBRT Comparator & $\begin{array}{l}\text { Payer perspective } \\
\mathbf{\$ 5 0 , 0 0 0 / Q A L Y}\end{array}$ & $\begin{array}{l}\text { Societal perspective } \\
\mathbf{\$ 5 0 , 0 0 0 / Q A L Y}\end{array}$ \\
\hline IMRT & $75.1 \%$ & $75.1 \%$ \\
PT & $94.1 \%$ & $95.5 \%$ \\
\hline
\end{tabular}

IMRT, intensity-modulated radiation therapy; $P T$, proton beam therapy; $Q A L Y$, quality-adjusted life year; SBRT, stereotactic body radiation therapy.

Table 8 | Alternative sensitivity analysis with proton therapy toxicity set equal to SBRT toxicity.

\begin{tabular}{|c|c|c|c|c|c|}
\hline & \multicolumn{2}{|c|}{ Total per patient } & \multicolumn{2}{|c|}{ Incremental } & \multirow{2}{*}{$\begin{array}{l}\text { ICER Costs/ } \\
\text { QALY gained }\end{array}$} \\
\hline & Costs & QALYs & Costs & QALYs & \\
\hline \multicolumn{6}{|c|}{ PAYER PERSPECTIVE } \\
\hline SBRT & $\$ 24,873$ & 8.11 & - & - & Reference \\
\hline IMRT & $\$ 33,068$ & 8.05 & $\$ 8,195$ & -0.062 & Weakly dominated* \\
\hline PT & $\$ 69,094$ & 8.17 & $\$ 44,221$ & 0.057 & $\$ 13,755,207$ \\
\hline \multicolumn{6}{|c|}{ SOCIETAL PERSPECTIVE $^{\dagger}$} \\
\hline SBRT & $\$ 25,097$ & 8.11 & - & - & Reference \\
\hline IMRT & $\$ 35,088$ & 8.05 & $\$ 9,991$ & -0.062 & Weakly dominated \\
\hline PT & $\$ 71,339$ & 8.17 & $\$ 46,242$ & 0.057 & $\$ 14,383,693$ \\
\hline
\end{tabular}

ICER, incremental cost-effectiveness ratio; IMRT, intensity-modulated radiation therapy; PT, proton beam therapy; $Q A L Y$, quality-adjusted life year; SBRT, stereotactic body radiation therapy.

NOTE: Results are estimated for a patient 65 years old using Medicare reimbursement rates.

*Dominated - higher cost and lower QALY.

${ }^{+}$Societal perspective also includes productivity costs owing to time spent in treatment.

compared to SBRT at a $\$ 100,000 /$ QALY threshold when all three toxicity rates for SBRT were increased by $54 \%$ from the base-case rates, from a payer's perspective. Similarly, from a societal perspective, IMRT was only found to be cost-effective at a $\$ 100,000 /$ QALY threshold when the toxicity rates of SBRT were increased by at least $61 \%$ from the base-case rates.

Among the three toxicities included in the model, the SD toxicity rate for SBRT was found to be lower than for IMRT. If the SD toxicity rate for SBRT is increased by more than $43 \%$ from the basecase rate, then the QALY for SBRT was lower than for IMRT. From a payer's perspective, the threshold analyses showed that IMRT was only cost-effective compared to SBRT at a \$100,000/QALY threshold when the SD toxicity rate for SBRT was increased by $97 \%$ from the base-case rate. Similarly, from a societal perspective, IMRT was only cost-effective compared to SBRT at a \$100,000/QALY threshold when the SD toxicity rate for SBRT was increased by $109 \%$ from the base-case rate.

\section{DISCUSSION}

In this study, we found that SBRT was less expensive than both IMRT and PT, with SBRT patients expecting to have a better quality of life owing to its more favorable toxicity profile. The 

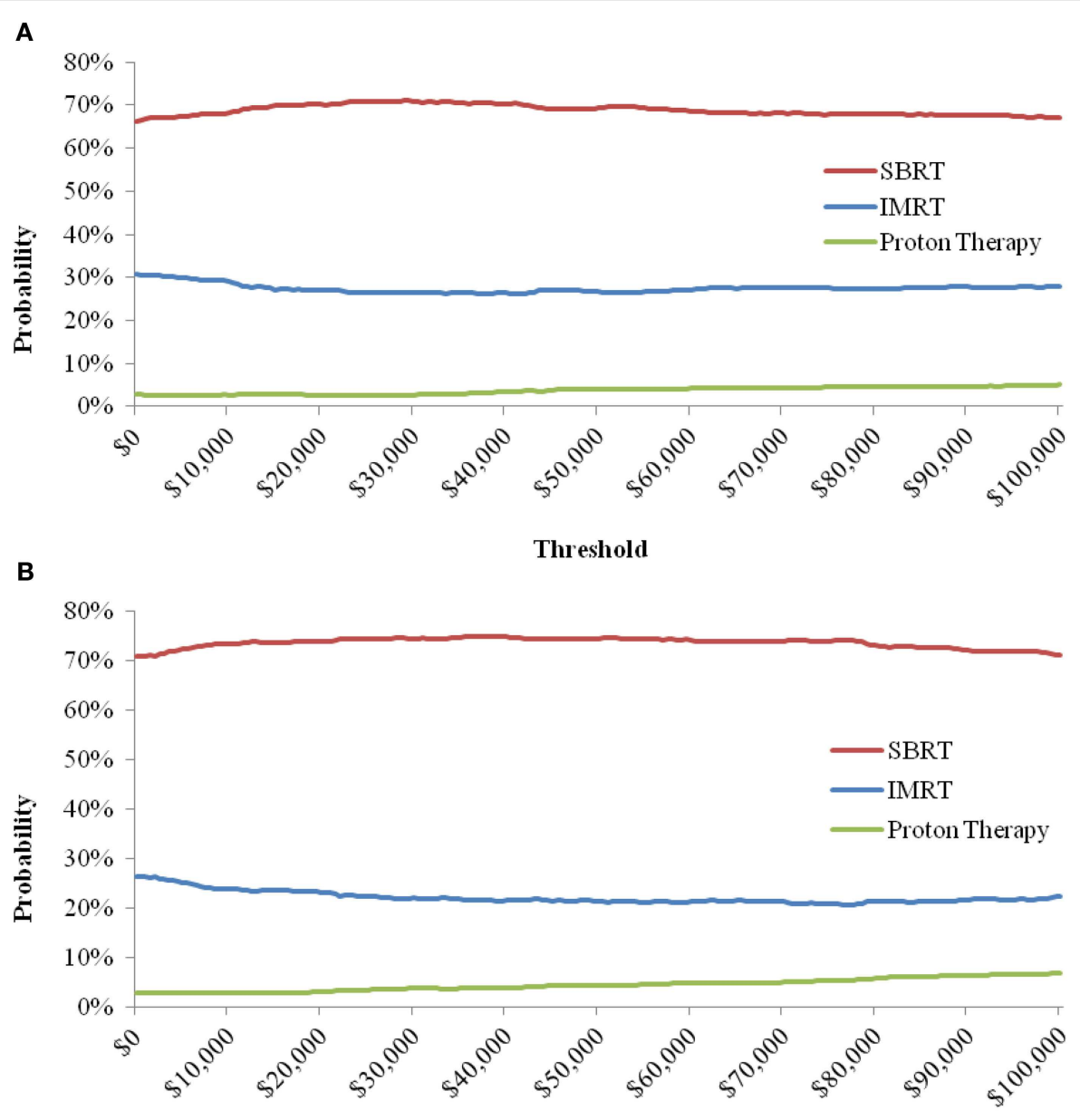

Threshold

FIGURE 2 | Probability of SBRT being cost-effective when compared to IMRT and Proton Therapy, from the Payer (A) and Societal (B) Perspective. Note: the cost-effectiveness acceptability curve shows the percentage of iterations in the probabilistic sensitivity analysis that are cost-effective at a certain threshold.

probabilistic sensitivity analyses from both the payer and societal perspectives showed that, compared to the other treatments, SBRT is cost-effective at a $\$ 50,000 /$ QALY threshold in $75 \%-96 \%$ of model simulations.

There is a continued trend of increasing utilization of more costly treatments for localized prostate cancer. From 2000 to 2008, the use of IMRT over the older 3D conformal radiation has increased from $28.7 \%$ to $81.7 \%$ (Nguyen et al., 2011). Since 2008 , there has been an exponential increase in the number of proton facilities built in the United States - each of which costs approximately $\$ 200$ million - with a corresponding increase in its use for prostate cancer treatment. Cost-effectiveness is one way to examine the "value" of different treatment options. Consistent with prior findings by Konski et al. (2007), we also demonstrate that proton therapy is unlikely to be cost-effective. Importantly, we find that newer technology does not always equate to costlier treatment. SBRT, because of an ability to shorten radiation treatment to five fractions, results in cost savings compared to IMRT and PT. If longer-term follow-up continues to demonstrate favorable toxicity and disease control outcomes for SBRT, then this study provides important data which may have policy implications.

This study has several limitations worth mentioning. There are limited data on SBRT for localized prostate cancer. In fact, since this study began, reports from five additional SBRT studies, each with varying numbers of patients and months of follow-up, have been published (Aluwini et al., 2010; Bolzicco et al., 2010; Kang et al., 2011; Townsend et al., 2011; Jabbari et al., 2012). None of these studies reached conclusions that differ substantially from the assumptions used in the present analysis (i.e., toxicity remains relatively low and disease control mirrors that obtained with other radiation therapy options). Further, the published literature on SBRT of localized prostate cancer has consisted mostly of patients treated with the CyberKnife Robotic Radiosurgery System (Accuray Incorporated, Sunnyvale, CA, USA; Friedland et al., 2009; King et al., 2009, 2012; Katz et al., 2010; Freeman and King, 2011). Two studies have been published which used conventional linear accelerators to deliver prostate SBRT (Madsen et al., 2007; Boike et al., 2011), but both used lower doses compared to current SBRT standards and therefore were not included in the model estimates. 
Inclusion of toxicity results from studies that used lower doses of SBRT may bias our results further in favor of SBRT. There is also a lack of data on the median time to resolution of long-term toxicity. Therefore, in the model, patients who develop long-term toxicity were assumed to remain in a health state reflective of their toxicity until they die.

In conclusion, based on the assumption that each treatment modality results in equivalent long-term disease control, results from this study suggest that SBRT is cost-effective, resulting in cost savings and improved quality-adjusted survival compared to IMRT and PT for the treatment of localized prostate cancer. Additional studies are needed to directly examine the comparative effectiveness of the different radiation treatments for localized prostate cancer. Given the lack of randomized trial data, this study provides important and novel information based on currently available published evidence, and contributes to an understanding of the comparative value of three

\section{REFERENCES}

Agency for Healthcare Research and Quality. (2010). Comparative Evaluation of Radiation Treatments for Clinically Localized Prostate Cancer: An Update. Technology Assessment Report ID: CANT1209.

Altekruse, S. F., Kosary, C. L., Krapcho, M., Neyman, N., Aminou, R., Waldron, W., Ruhl, J., Howlader, N., Tatalovich, Z., Cho, H., Mariotto, A., Eisner, M. P., Lewis, D. R., Cronin, K., Chen, H. S., Feuer, E. J., Stinchcomb, D. G., and Edwards, B. K. (eds). (2010). SEER Cancer Statistics Review, 1975-2007. Bethesda, MD: National Cancer Institute.

Aluwini, S., van Rooij, P., Hoogeman, M., Bangma, C., Kirkels, W. J., Incrocci, L., and Kolkman-Deurloo, I. K. (2010). Cyber knife stereotactic radiotherapy as monotherapy for low- to intermediate-stage prostate cancer: early experience, feasibility, and tolerance. J. Endourol. 24, 865-869.

Boike, T. P., Lotan, R., Cho, L. C., Brindle, J., DeRose, P., Xie, X. J., Yan, J., Foster, R., Pistenmaa, D., Perkins, A., Cooley, S., and Timmerman, R. (2011). Phase I dose-escalation study of stereotactic body radiation therapy for low- and intermediaterisk prostate cancer. J. Clin. Oncol. 29, 2020-2026.

Bolzicco, G., Favretto, M. S., Scremin, E., Tambone, C., Tasca, A., and Guglielmi, R. (2010). Image-guided stereotactic body radiation therapy for clinically localized prostate cancer: preliminary clinical results. Technol. Cancer Res. Treat. 9, 473-477.

Bureau of Labor Statistics. (2011). Usual Weekly Earnings of Wage and Salary
Workers, ed. M. D. Levi (Bureau of Labor Statistics).

Chen, R. C., Clark, J. A., and Talcott, J. A. (2009). Individualizing qualityof-life outcomes reporting: how localized prostate cancer treatments affect patients with different levels of baseline urinary, bowel, and sexual function. J. Clin. Oncol. 27, 3916-3922.

Coen, J. J., Paly, J. J., Niemierko, A., Weyman, E., Rodrigues, A., Shipley, W. U., Zietman, A. L., and Talcott, J. A. (2012). Long-term quality of life outcome after proton beam monotherapy for localized prostate cancer. Int. J. Radiat. Oncol. Biol. Phys. 82, e201-e209.

Cooke, C. E., Wong, W., and Lee, H. (2005). Utilization and cost of sildenafil in a large managed care organization with a quantity limit on sildenafil. J. Manag. Care Pharm. 11, 674-680.

Freeman, D. E., and King, C. R. (2011). Stereotactic body radiotherapy for low-risk prostate cancer: five-year outcomes. Radiat. Oncol. 6, 3.

Friedland, J. L., Freeman, D. E., Masterson-McGary, M. E., and Spellberg, D. M. (2009). Stereotactic body radiotherapy: an emerging treatment approach for localized prostate cancer. Technol. Cancer Res. Treat. 8, 387-392.

Hamner, J., Lawrence, W. F., Anderson, J. P., Kaplan, R. M., and Fryback, D. G. (2006). Report of nationally representative values for the noninstitutionalized US adult population for 7 health-related quality-oflife scores. Med. Decis. Making 26, 391-400.

Jabbari, S., Weinberg, V. K., Kaprealian, T., Hsu, I. C., Ma, L., Chuang,

types of external beam radiation treatments for localized prostate cancer.

\section{AUTHORS' CONTRIBUTIONS}

Anju Parthan: Primary investigator, Narin Pruttivarasin: Primary economic modeler, Douglas C. A. Taylor: Study director, Vivek Pawar: Secondary economic modeler, Diane Davies: Health economist and author, Akash Bijlani: Health policy/economic specialist and author, Kristen Hassmiller Lich: Health policy specialist and author, Ronald Chen: Radiation oncologist and senior author.

\section{ACKNOWLEDGMENTS}

We would like to thank Grace Yang, MPA, MA, for her help with conducting the meta-analysis. We would also like to thank Virginia $\mathrm{M}$. Rosen, $\mathrm{PhD}$, who provided medical writing assistance on behalf of OptumInsight which was contracted by Accuray Incorporated to produce this manuscript.

C., Descovich, M., Shiao, S., Shinohara, K., Roach, M. III, and Gottschalk,A. R. (2012). Stereotactic body radiotherapy as monotherapy or post-external beam radiotherapy boost for prostate cancer: technique, early toxicity, and PSA response. Int. J. Radiat. Oncol. Biol. Phys. 82, 228-234.

Kang, J. K., Cho, C. K., Choi, C. W. Yoo, S., Kim, M. S., Yang, K., Yoo, H., Kim, J. H., Seo, Y. S., Lee, D. H., and Jo, M. (2011). Image-guided stereotactic body radiation therapy for localized prostate cancer. Tumori 97, 43-48.

Katz, A. J., Santoro, M., Ashley, R., Diblasio, F., and Witten, M. (2010). Stereotactic body radiotherapy for organconfined prostate cancer. BMC Urol. 10, 1. doi:10.1186/1471-2490-10-1

King, C. R., Brooks, J. D., Gill, H. Pawlicki, T., Cotrutz, C., and Presti, J. C. Jr. (2009). Stereotactic body radiotherapy for localized prostate cancer: interim results of a prospective phase II clinical trial. Int J. Radiat. Oncol. Biol. Phys. 73, 1043-1048.

King, C. R., Brooks, J. D., Gill, H. and Presti, J. C. Jr. (2012). Longterm outcomes from a prospective trial of stereotactic body radiotherapy for low-risk prostate cancer. Int. J. Radiat. Oncol. Biol. Phys. 82, 877882.

Kirichenko, A. V., Ruth, K., Horwitz, E. M., Buyyounouski, M. K., Feigenberg, S. J., Chen, D. Y., and Pollack, A. (2006). Intensity modulated radiation therapy for prostate cancer: preliminary results on treatment morbidity compared to 3$\mathrm{D}$ conformal radiation therapy. Int. J. Radiat. Oncol. Biol. Phys. 66, S326.
Konski, A., Speier, W., Hanlon, A., Beck, J. R., and Pollack, A. (2007). Is proton beam therapy cost effective in the treatment of adenocarcinoma of the prostate? J. Clin. Oncol. 25, 3603-3608.

Konski, A., Watkins-Bruner, D., Feigenberg, S., Hanlon, A., Kulkarni, S., Beck, J. R., Horwitz, E. M., and Pollack, A. (2006). Using decision analysis to determine the costeffectiveness of intensity-modulated radiation therapy in the treatment of intermediate risk prostate cancer. Int. J. Radiat. Oncol. Biol. Phys. 66, 408-415.

Madsen, B. L., His, R. A., Pham, H. T., Fowler, J. F., Esagui, L., and Corman, J. (2007). Stereotactic hypofractionated accurate radiotherapy of the prostate (SHARP), 33.5 Gy in five fractions for localized disease: first clinical results. Int. J. Radiat. Oncol. Biol. Phys. 67, 1009-1105.

MAG Mutual. (2011). 2011 MAG Mutual Physicians' Fee and Coding Guide. Atlanta: MAG Mutual Healthcare Solutions, Inc., 2010. Print.

Martin, A., and Gaya, A. (2010). Stereotactic body radiotherapy: a review. Clin. Oncol. 22, 157-172.

National Cancer Institute. (2012). Bethesda, MD: NIH, DHHS. Available at: http://www.cancer.gov/ cancertopics/types/prostate [accessed May 24, 2012].

Nguyen, P. L., Gu, X., Lipsitz, S. R. Choueiri, T. K., Choi, W. W., Lei, Y., Hoffman, K. E., and Hu, J. C. (2011). Cost implications of the rapid adoption of newer technologies for treating prostate cancer. J. Clin. Oncol. 29, 1517-1524 
Ollendorf, D. A., Hayes, J., McMahon, P., Kuba, M., Tramontano, A., and Pearson, S. D. (2008). Brachytherapy \& Proton Beam Therapy for Treatment of Clinically-Localized, LowRisk Prostate Cancer. Boston: Institute for Clinical and Economic Review. [Final appraisal document].

Ollendorf, D. A., Hayes, J., McMahon, P., and Pearson, S. D. (2009). Active Surveillance \& Radical Prostatetomy for the Management of Low-Risk, Clinically-Localized Prostate Cancer. Boston: Institute for Clinical and Economic Review. [Final appraisal document].

Red Book. (2010). Pharmacy's Fundamental Reference. Montvale, NJ: Thomson Reuters (Healthcare) Inc.

Sanda, M. G., Dunn, R. L., Michalski, J., Sandler, H. M., Northouse, L., Hembroff, L., Lin, X., Greenfield, T. K., Litwin, M. S., Saigal, C. S., Mahadevan, A., Klein, E., Kibel, A., Pisters, L. L., Kuban, D., Kaplan, I., Wood, D., Ciezki, J., Shah, N., and Wei, J. T. (2008). Quality of life and satisfaction with outcome among prostate-cancer survivors. N. Engl. J. Med. 358, 1250-1261.

Schulte, R. W., Slater, J. D., Rossi, C. J. Jr., and Slater, J. M. (2000). Value and perspectives of proton radiation therapy for limited stage prostate cancer. Strahlenther. Onkol. 176, 3-8.

Slater, J. D., Rossi, C. J. Jr., Yonemoto, L. T., Reyes-Molyneux, N. J., Bush, D. A., Antoine, J. E., Miller, D. W., Teichman, S. L., and Slater, J. M. (1999). Conformal proton therapy for earlystage prostate cancer. Urology 53, 978-984.

Slater, J. D., Yonemoto, L. T., Rossi, C. J. Jr., Reyes-Molyneux, N. J., Bush, D. A., Antoine, J. E., Loredo, L. N., Schulte, R. W., Teichman, S. L., and Slater, J. M. (1998). Conformal proton therapy for prostate carcinoma. Int. J. Radiat. Oncol. Biol. Phys. 42, 299-304.

Stewart, S. T., Lenert, L., Bhatnagar, V., and Kaplan, R. M. (2005). Utilities for prostate cancer health states in men aged 60 and older. Med. Care 43, 347-355.

Townsend, N. C., Huth, B. J., Ding, W., Garber, B., Mooreville, M.
Arrigo, S., Lamond, J., and Brady, L. W. (2011). Acute toxicity after cyberknife-delivered hypofractionated radiotherapy for treatment of prostate cancer. Am. J. Clin. Oncol. 34, 6-10.

Wiegner, E. A., and King, C. R. (2010). Sexual function after stereotactic body radiotherapy for prostate cancer: results of a prospective clinical trial. Int. J. Radiat. Oncol. Biol. Phys. 78, 442-448.

Zelefsky, M. J., Chan, H., Hunt, M., Yamada, Y., Shippy, A. M., and Amols, H. (2006). Long-term outcome of high dose intensity modulated radiation therapy for patients with clinically localized prostate cancer. J. Urol. 176(4 Pt 1), 1415-1419.

Zelefsky, M. J., Fuks, Z., Hunt, M. Yamada, Y., Marion, C., Ling, C. C., Amols, H., Venkatraman, E. S. and Leibel, S. A. (2002). High-dose intensity modulated radiation therapy for prostate cancer: early toxicity and biochemical outcome in 772 patients. Int. J. Radiat. Oncol. Biol. Phys. 53, 1111-1116.
Conflict of Interest Statement: This study was funded by Accuray, Inc.

Received: 20 June 2012; paper pending published: 04 July 2012; accepted: 12 July 2012; published online: 20 August 2012. Citation: Parthan A, Pruttivarasin N, Davies D, Taylor DCA, Pawar V, Bijlani A, Lich KH and Chen RC (2012) Comparative cost-effectiveness of stereotactic body radiation therapy versus intensitymodulated and proton radiation therapy for localized prostate cancer. Front. Oncol. 2:81. doi: 10.3389/fonc.2012.00081

This article was submitted to Frontiers in Radiation Oncology, a specialty of Frontiers in Oncology.

Copyright (c) 2012 Parthan, Pruttivarasin, Davies, Taylor, Pawar, Bijlani, Lich and Chen. This is an open-access article distributed under the terms of the Creative Commons Attribution License, which permits use, distribution and reproduction in other forums, provided the original authors and source are credited and subject to any copyright notices concerning any third-party graphics etc. 